\title{
Recent-onset of rheumatoid arthritis leads to increase in wall thickness of left anterior descending coronary artery
}

\author{
An evidence of subclinical coronary artery disease
}

Suad Hannawi, MSc, PhD, Haifa Hannawi, MSc, PhD, Fahda Alokaily, MSc, PhD, Esperanza Naredo, MSc, PhD, Ingrid Moller, MSc, PhD, Issa Al Salmi, MSc, PhD.

\begin{abstract}

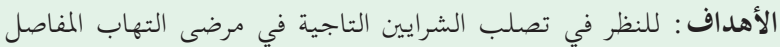

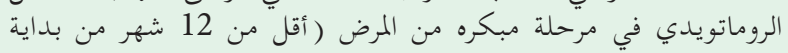
(الأعراض ) ( الروماتري

الطريقة : تم إجراء فحص أشعة صوتية للقلب على 15 من من مرضى الروماتويد

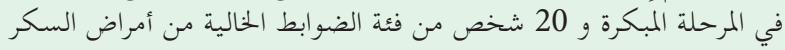

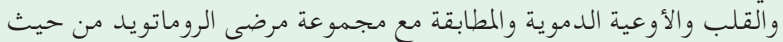

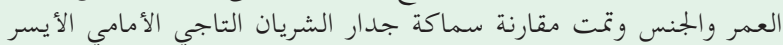

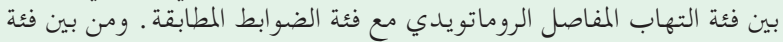

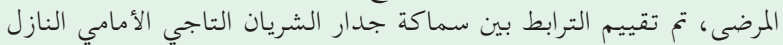

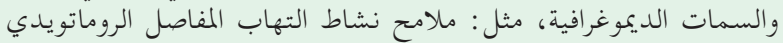

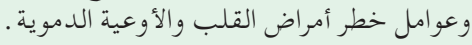

النتائح: زادت لدى مرضى التهاب المفاصل الروماتويدي في المرحلة المبكرة

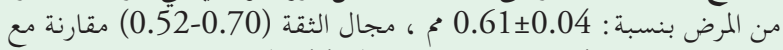

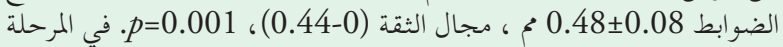

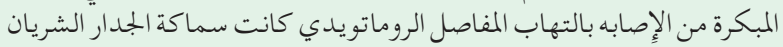

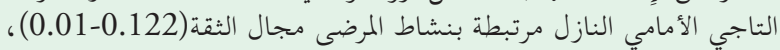

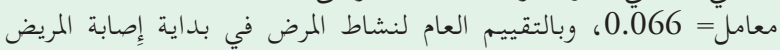

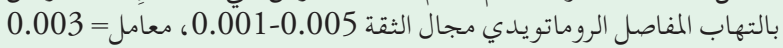

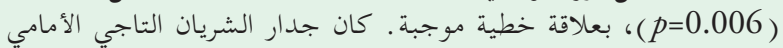

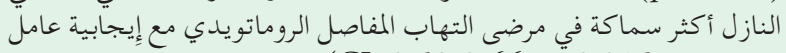

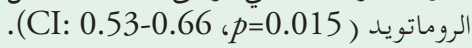

الخاتمة: المرضى المصابين بالتهاب المفاصل الروماتويدي في المرحلة المبكرة

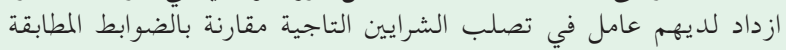

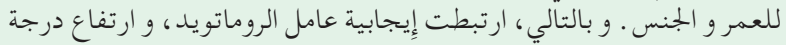

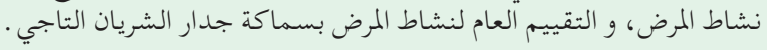

Objectives: To explore the atherosclerotic changes of the left anterior descending coronary artery (LADCA) in patients with early onset of rheumatoid arthritis (RA).

Methods: Transthoracic echocardiographic scans were performed on 15 early RA patients and 20 control subjects, free of rheumatological diseases, diabetes mellitus (DM), and cardiovascular disease (CVD). Out of 15 RA patients, 10 were matched for age and gender with control. Left anterior descending coronary artery wall thickness was compared between RA subjects and their matched control. Among early RA patients, correlation was assessed between LADCA wall thickness and the demographic features, RA activity features, and cardiovascular risk factors.

Results: Left anterior descending coronary artery wall thickness was significantly increased $(p=0.001)$ in early RA compared to controls, as it was reported to be $0.61 \pm 0.04$ $\mathrm{mm}$ (CI: $0.52-0.7)$; and $0.48 \pm 0.08 \mathrm{~mm}$ (CI: 0.44-0.51) respectively. Within early RA, LADCA wall thickness was related to the disease activity score $(p=0.025$, $B$-coefficient 0.066 , CI: $0.01-0.122$ ) as well as to the patient global assessment of disease activity (PGADA) at RA onset $(p=0.006$, ß-coefficient 0.003, CI: 0.001-0.005), in a positive linear relationship. Left anterior descending coronary artery wall thickness was found to be thicker among RA patients with rheumatoid factor (RF) positive ( $p=0.015$, CI: 0.53-0.66).

Conclusion: Early RA patients have increased coronary arteries atherosclerotic burden compared to healthy subjects matched for age, and gender. Rheumatoid factor positivity, high disease activity score and PGADA were found to be associated with coronary artery wall thickness.

Saudi Med J 2018; Vol. 39 (12): 1213-1217 doi: 10.15537/smj.2018.12.23185

From the Ministry of Health and Prevention (Hannawi, Hannawi), Dubai, United Arab Emirates, from the Rheumatology Department (Alokaily), Prince Sultan Military Medical City, Riyadh, Kingdom of Saudi Arabia; Department of Rheumatology (Naredo), Joint and Bone Research Unit, Hospital Universitario Fundación Jiménez Díaz and Autónoma University, Madrid; from the Instituto Poal de Reumatologia (Moller), Barcelona, Spain; and the Royal Hospital (Al Slami), Muscat, Oman.

Received 19th June 2018. Accepted 5th November 2018.

Address correspondence and reprint request to: Associate Professor Suad Hannawi, Ministry of Health and Prevention, Dubai, United Arab Emirates.E-mail: suad1@ausdoctors.net

ORCID ID: orcid.org/0000-0002-5689-6710 
$\mathrm{R}^{\mathrm{h}}$ heumatoid arthritis (RA) is a chronic inflammatory and destructive joint disease affecting around 1-3\% of the general population and is associated with increased mortality and morbidity with cardiovascular disease (CVD) as an underlying leading cause. ${ }^{1}$ The role of inflammation has been well established in the formation, progression, and complication of atherosclerosis in RA. ${ }^{2}$ Ultrasound (US) testing has provided valuable insights into early atherogenesis by measuring carotid intimamedia thickness, ${ }^{3,4}$ and endothelial dysfunction (ED), and these factors are the indicator of atherosclerosis at the earliest stage. ${ }^{5,6}$ It has been demonstrated that early and established RA patients have increased risk of sub-clinical vascular disease compared to their matched controls due to the high prevalence of carotid disease ${ }^{5,7}$ and $\mathrm{ED} .{ }^{6,8}$ Atherosclerosis is considered to be a diffuse process, and it can be evaluated in other vessels such as the left anterior descending coronary artery (LADCA). ${ }^{9}$ Therefore, we investigated, whether the presence of generalized atherosclerotic changes are evident at early RA by studying LADCA wall thickness in RA patients presenting within 12 months of symptom onset.

Left anterior descending coronary artery and its proximal branches have been identified with 2-dimensional transthoracic echocardiography (TDTE) using short axis views. ${ }^{10-13}$ Left anterior descending coronary artery wall thickness was found to be significantly increased in coronary artery disease patients compared to healthy subjects. Furthermore, the high-resolution TDTE was found to be sensitive enough to detect these differences. ${ }^{14}$

Methods. Study participants. All 15 RA study participants including 7 female (47\%), and 8 male (53\%) met the ACR 1987 revised criteria for the classification of RA. ${ }^{15}$ All the patients had RA symptoms for less than 12 months. None of the patients were on non-steroidal anti-inflammatory drugs (NSAIDs). Twelve out of the 15 RA patients were on methotrexate only, 2 were on methotrexate and sulfasalazine, and one was on a combination of methotrexate, sulfasalazine, and hydroxychloroquine. None of the patients were on biological medications. Twenty healthy subjects were recruited from the community. The controls matched with 10 early RA patients (out of total 15) for age, gender, and cardiovascular risk factors. All the participants were free of $\mathrm{CVD} /$ cerebrovascular disease (CBD), and DM.

Disclosure. Authors have no conflict of interests, and the work was not supported or funded by any drug company. Dr. Fahda Alokaily is a member of the Editorial Team, and was therefore excluded from any final editorial decisions regarding this paper.
Study procedure. The study was approved by the Research Ethics Committee of the Ministry of Health and Prevention of United Arab Emirates. All subjects provided written informed consent. Participants histories related to cardiovascular risk factors, smoking, CVD/CBD, DM, hypertension, dyslipidemia, family history of vascular events in first degree relatives, and body mass index were recorded.

Rheumatoid arthritis disease activity measurements. The participants were examined for the presence of RA nodules, RF positivity and level, anti CCP positivity and level, Disease Activity Score based on 4 variable using erythrocyte sedimentation rate (ESR) and C-reactive protein (CRP), health assessment questionnaire score, PGADA (DAS 28 with 4 variable), joints pain, and number of tenders and swollen joints based on 28 joints count.

Lipid profile measurements. Laboratory parameters including low-density lipoprotein, high-density lipoprotein, triglycerides, and cholesterol level were measured for all the participants.

Left anterior descending coronary artery wall thickness measurements. Left anterior descending coronary artery wall thickness was measured soon after the confirmed diagnosis of RA with no more than 12 months of symptoms onset. The transthoracic images were obtained using the Philips iE33 (Andover, MA). A single operator carried out all transthoracic echocardiographic measurements. Patients were examined in the dorsal decubitus position. Transthoracic echocardiographic images were obtained with the $3 \mathrm{MHz}$ phased array transducer. The left anterior descending coronary artery was viewed in the parasternal short axis plane. Coronary arteries appeared as linear tubular structure. The wall thickness was measured off-line by expert echocardiographers (Figure 1). The LADCA wall thickness measurements were obtained from the outer edge to the inner edge of the line representing the vascular wall. The reader was blinded to any clinical data and was free to choose any frames that he believed well visualized the LADCA wall. All measurements were obtained on 3 frames, and the results were averaged. Clear images were obtained in all the study participants. There were no significant inter $(p=0.89)$ or intra-observer $(p=0.30)$ variability in LADCA wall thickness readings.

Statistical analysis. Continuous variables were tested for normality of distribution. Transformations were applied for non-normally distributed variables. Differences between the LADCA wall thickness in RA patients and the matched control group were analyzed by 2-sample (independent) Student's t-test. Association of LADCA wall thickness with age, continuous $\mathrm{CV}$ variables, and disease activity variables were evaluated using linear regression analysis. The 2 -sided probability 


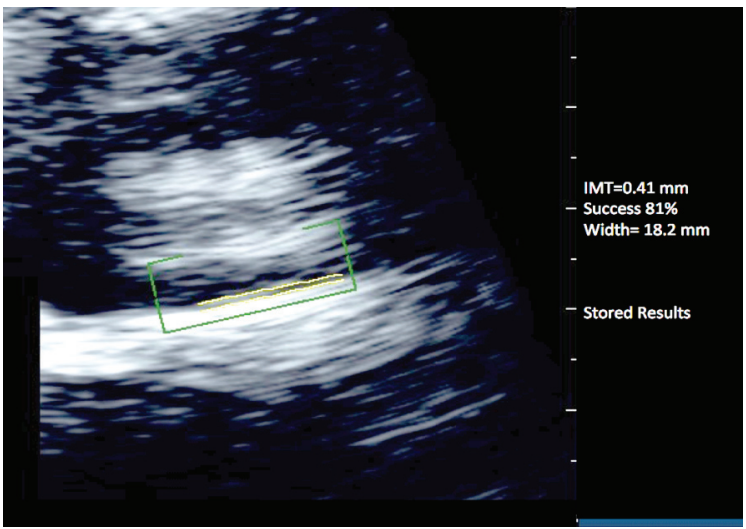

Figure 1 - Coronary arteries appeared as linear tubular structure. The wall thickness was measured off-line.

values of $p<0.05$ were considered statistically significant. For all the analysis, Stata 10/SE statistical software (Stata Corp, Texas, USA) was used.

Results. Clinical features. Fifteen RA patients and 20 control subjects with matched 10 RA participants were included in the study. Demographic details, RA characteristics, and laboratory values of 15 RA patienty at first presentation are shown in Table 1. None of the RA patients or the control participants had previous CVD/CBD or DM. There were no significant differences in the history of ever smoking, current smoking, hypertension, hyperlipidemia, or family history of CVD/CBD between the 2 groups. Systolic and diastolic blood pressure, level of cholesterol, triglyceride, HDL, LDL, and cholesterol levels between control participants and RA patients, revealed no significant difference (results not shown). Among RA patients, the mean age at the time of diagnosis was 54 (26-78 years), with no significant difference in age between males $(57 \pm 13.6$ years $)$ and females $(51 \pm 16$ years). The mean duration of RA symptoms at the time of scanning was $2 \pm 4$ (range 1-12) months.

Left anterior descending artery wall thickness in $R A$ patients and their matched controls. The LADCA wall thickness was measured in all early RA patients and control subjects. Despite the fact, that the cardiovascular risk factors did not differ significantly between RA patients and control group, however, the LADCA wall thickness was significantly increased $(\mathrm{p}=0.001)$ in patients with early RA $(0.61 \pm 0.04$ $\mathrm{mm}$, CI: 0.52-0.70), than among their matched controls $(0.48 \pm 0.08 \mathrm{~mm}, \mathrm{CI}: 0.44-0.51)$, (Figure 2). Within the 15 early RA patients, univariate modeling revealed that LADCA wall thickness was related to Disease Activity Score ( $p=0.025$, ß-coefficient 0.066, CI: $0.010-0.122$ ) and to the PGADA at RA onset ( $p=0.006$, B-coefficient 0.003, CI: $0.001-0.005)$, in positive linear relationship. LADCA wall thickness was
Table 1 - Demographic details, RA characteristics, and laboratory values of 15 RA patients at first presentation.

\begin{tabular}{lc}
\hline Demographic details & n (\%) \\
\hline Male & 8 \\
Female & 7 \\
Mean age (range, year) & $54(26-78)$ \\
Male mean age (range, year) & $57(37-78)$ \\
Female mean age (range, year) & $51(26-69)$ \\
Body mass index (range, kg/m ${ }^{2}$ ) & $28(17-35)$ \\
Rheumatoid arthritis characteristics & \\
Disease duration mean (range, months) & $2.2(1-12)$ \\
Tender joint count (of 53) & $21(14.0)$ \\
Swollen joint count (of 44) & $20(8.0)$ \\
Health assessment questionnaire score & $3.3(3.5)$ \\
(maximum disability 24) & \\
Physician's global assessment of disease activity & $37(25.0)$ \\
(maximum 100) & \\
Joint pain (VAS, maximum 100) & 41 \\
Morning stiffness duration (minutes) & $150(20)$ \\
Rheumatoid factor level & $230(443)$ \\
Disease activity score (DAS-28) 4v & $5.0(1.5)$ \\
Rheumatoid factor positive, n (\%) & $9(60.0)$ \\
RA nodules, n (\%) & $1(7.0)$ \\
Other extra-articular features, n (\%) & 0 \\
Cardiovascular risk factors (\%) & \\
History of ever smoking & \\
History of current smoking & 1 \\
History of hypertension & $(6.0)$ \\
History of diabetes mellitus & $0(0.0)$ \\
History of hyperlipidemia & $2(13.0)$ \\
Family history of cardiovascular disease & $0(0.0)$ \\
Systolic blood pressure & $2(13.0)$ \\
Diastolic blood pressure & $0(0.0)$ \\
Laboratory values & $125(22.0)$ \\
ESR (mm/hr) & $75(12.0)$ \\
CRP (mg/L, normal <6) & \\
Haemoglobin (g/L) & $44(23.0)$ \\
Cholesterol (mmol/L) & $28(24.0)$ \\
Triglyceride (mmol/L) & $4.8(11.0)$ \\
HDL level (mmol/L) & $1.7(1.1)$ \\
LDL cholesterol (mmol/L) & $1.4(0.4)$ \\
\hline RA - rheumatoid arthritis, SD - standard deviation, VAS & $2.7(0.6)$ \\
scale, ESR - erythrocyte sedimentation rate, CRP - C-reactive protein, & \\
HDL - high density lipoprotein, LDL - low density lipoprotein \\
\hline
\end{tabular}

higher among RA patients with positive RF ( $p=0.015$, CI: 0.53 - 0.66), (Figure 3).

Discussion. These patients have higher chances of coronary arteries atherosclerotic problems compared to healthy subjects matched for age, gender and CVD risk factors. ${ }^{5}$ Atherosclerosis is a slowly progressive disease affecting coronary arteries as well as the large and medium-sized arteries. ${ }^{14}$ It has been demonstrated that changes in the size of the coronary artery lumen are not adequate for the diagnosis of subclinical coronary atherosclerosis because the disease is considered to be present in the arterial wall before luminal changes are evident. ${ }^{15}$ Therefore, detecting the progression of atherosclerosis requires an evaluation of variations in the coronary arterial wall thickness. ${ }^{14}$ Transthoracic echocardiography has been demonstrated to be a valid, feasible and reliable technique for measurement of distal LADCA diameter, ${ }^{16}$ and coronary wall thickness. ${ }^{16,17}$ 


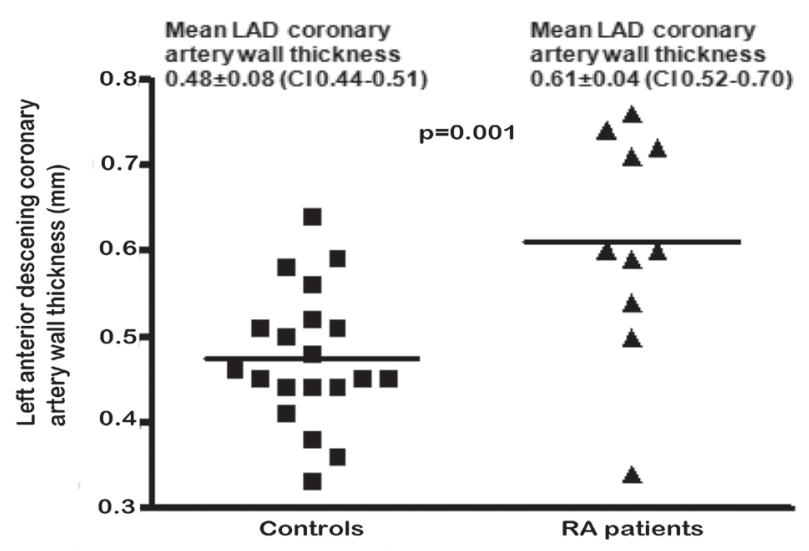

Figure 2 - Left anterior descending coronary artery wall thickness (mm) in rheumatoid arthritis (RA) patients versus control group.

The distal portion of the LADCA positioned close to the chest wall ${ }^{16}$ facilitates the scanning process. Furthermore, since coronary artery disease is a diffuse process, and the LADCA is the most frequently involved vessel, the coronary segment we scanned should represent an adequate sample of the coronary vasculature for the detection of subclinical atherosclerosis. Such noninvasive technique would permit the early detection of coronary atherosclerosis, and consequently, reducing the unfortunate coronary events, even in asymptomatic RA patients. ${ }^{18}$ The previous study showed that the standard LADCA thickness is $0.357 \pm 0.06 \mathrm{~mm}$ for males and $0.25 \pm 0.03 \mathrm{~mm}$ for females. ${ }^{19}$ Moreover, the LADCA thickness could be as high as $0.78 \pm 0.3$ $\mathrm{mm}$ in patients with coronary atherosclerosis. ${ }^{20}$ The mean LADCA thickness in this study was found to be $0.48 \pm 0.08 \mathrm{~mm}$ for healthy control and $0.61 \pm$ $0.04 \mathrm{~mm}$ for early RA patients. Differences between our measurements and any other study using the same techniques could be justified by presence of inflammatory activity in RA patients and population characteristics of the participants. In addition to this the LADCA wall thickness exhibited a significant correlation to the Disease Activity Score, PGADA, and to RF positivity. Furthermore, the variation in readings may also be due to the difference of recording site, or the transducer frequency. Unfortunately, we did not find any previously published report regarding LADCA wall thickness measurement in early RA patients to compare our outcomes. In addition to LAD coronary intima US, other new cardiovascular risk imaging techniques are continually being proposed, such as computed tomography (CT) and coronary magnetic resonance (CMR). CT can be used for coronary artery calcium score (CACS) or coronary angiography (CA). It has been reported that patients with long-term RA have coronary artery calcification which is associated with inflammatory activity. ${ }^{19}$ Although it has been suggested that CACS is a simple test to estimate the

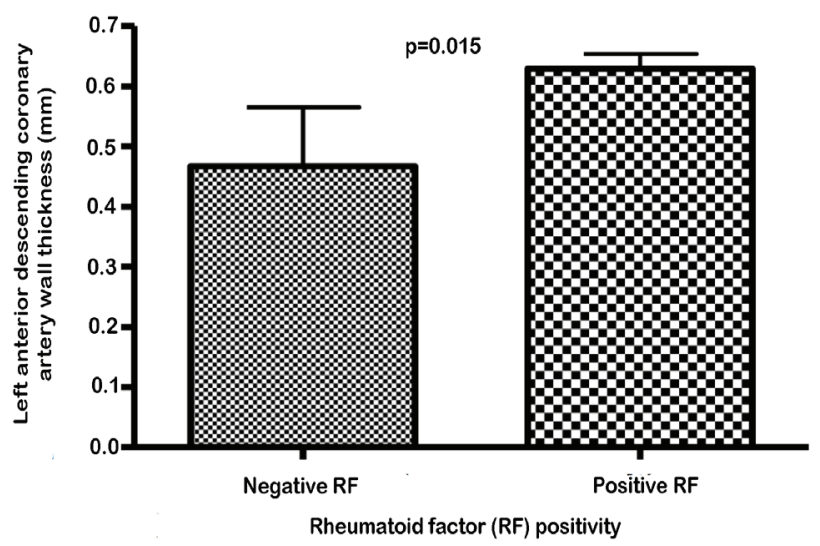

Figure 3 - Left anterior descending coronary artery wall thickness (mm) in rheumatoid factor (RF) positive rheumatoid arthritis (RA) patients versus RF negative RA patients.

degree of calcification within the coronary arteries and it is a direct measure of early atherosclerosis. ${ }^{20} \mathrm{~A}$ more recent study suggests that some patients identified as low risk with traditional scoring methods would fall into higher risk categories with coronary artery calcium scoring. ${ }^{21}$ Others found that high CACS can modify predicted risk obtained from Framingham scores alone, especially among patients in the intermediate-risk category in whom clinical decision making is most uncertain. $^{22}$ Although increasing CACS content is generally predictive of a higher likelihood of ischemia, its absence does not eliminate the possibility of flowlimiting coronary artery disease. ${ }^{23}$ On the other hand, CT requires the administration of iodinated contrast medium that might be associated with intolerance symptoms or renal impairment. Furthermore, the patients are exposed to ionizing radiation which is higher than in invasive CA. ${ }^{24}$ As a result, CMR angiography has been introduced to overcome these limitations. CMR is associated with non-invasive visualization of the epicardial coronary arteries. Generally, CMR has high sensitivity and overall accuracy for detecting coronary artery diseases. ${ }^{25}$ However, coronary arteries with diameters less than $1.5 \mathrm{~mm}$ are not well visualized by CMR angiography that results in an unsuccessful rate of $13 \%-14 \% .{ }^{26}$ Consequently, CMR has a lower diagnostic accuracy of distal coronary artery lesions than the CT. ${ }^{27}$ Other limitations of CMR include its expense and contraindications for patients with retained metal objects or old metallic medical prosthesis. Therefore, it is hard at this stage, with a limited data to compare LADCA thickness with the CACS or CMR but what favor LADCA thickness that it can be done in the clinic setting while the CACS test is usually done by a radiology technologist and is not an officebased technique. Nevertheless, with all the techniques LADCA, CTA and CMR presently have incomplete longitudinal prognostic data specific to RA patients to guide rheumatologist in using them in CVD risk 
stratification.

The strength of our study comes from matching controls for age and gender to RA patients without any comorbid conditions including $\mathrm{CVD} / \mathrm{CBD}$ and DM. Since CVD is a diffuse process and it rarely spares the proximal LADCA (Hausmann, Friedrich, et al. 1996 ). Therefore, this segment of coronary artery proved to be a good sampling site for the detection of subclinical coronary artery disease among RA population. Additionally, it has been verified that highresolution broadband transducer, TDTE have sufficient penetration and resolution to appraise the coronary artery wall thickness, ${ }^{20}$ that represent subclinical coronary atherosclerosis.

Our study has some limitations, as it involves only early RA patients, we cannot extrapolate our findings to all RA population. However, we showed that high-resolution transthoracic echocardiography is sufficiently sensitive to detect coronary atherosclerosis in early RA patients. Another limitation of the study is that it is difficult to distinguish that we have measured consecutively the same segment of the LADCA because of rotation and translational effects throughout the cardiac cycle. We tried to decrease this variability by maintaining the transducer in a fixed position throughout the scanning process.

This study supports our earlier published report about the presence of subclinical atherosclerotic changes among early RA patients. ${ }^{5}$ Furthermore, our results indicate that a transthoracic ultrasound examination of the LADCA could be a clinically valuable tool in the detection of subclinical coronary artery disease among RA patients.

In conclusion, this is the first study to demonstrate that LADCA wall thickness could be measured in early RA patients and these patients are at higher risk of developing coronary arteries atherosclerotic ailments compared to healthy subjects, matched for age, gender and cardiovascular risk factors.

\section{References}

1. Turiel M, Sitia S, Tomasoni L, Cicala S, Atzeni F, Gianturco $\mathrm{L}$, et al. [Cardiac involvement in rheumatoid arthritis]. Reumatismo 2009; 61: 244-253. Italian

2. Gabriel SE, Crowson CS. Risk factors for cardiovascular disease in rheumatoid arthritis. Curr Opin Rheumatol 2012; 24: 171-176.

3. Naredo E, Möller I, Gutiérrez M, Bong DA, Cobo T, Corominas $\mathrm{H}$, et al. Multi-examiner reliability of automated radio frequency-based ultrasound measurements of common carotid intima-media thickness in rheumatoid arthritis. Rheumatology (Oxford) 2011; 50: 1860-1864.

4. Naredo E, Möller I, Corrales A, Bong DA, Cobo-Ibáńez T, Corominas $\mathrm{H}$, et al. Automated radiofrequency-based US measurement of common carotid intima-media thickness in RA patients treated with synthetic vs synthetic and biologic DMARDs. Rheumatology (Oxford) 2013; 52: 376-381.

5. Hannawi S, Haluska B, Marwick TH, Thomas R. Atherosclerotic disease is increased in recent-onset rheumatoid arthritis: a critical role for inflammation. Arthritis Res Ther 2007; 9: R116.
6. Hannawi S, Marwick TH, Thomas R. Inflammation predicts accelerated brachial arterial wall changes in patients with recent-onset rheumatoid arthritis. Arthritis Res Ther 2009; 11: R51.

7. van Sijl AM, Peters MJ, Knol DK, de Vet HC, Gonzalez-Gay MA, Smulders YM, et al. Carotid intima media thickness in rheumatoid arthritis as compared to control subjects: a metaanalysis. Semin Arthritis Rheum 2011; 40: 389-397.

8. Vaudo G, Marchesi S, Gerli R, Allegrucci R, Giordano A, Siepi D, et al. Endothelial dysfunction in young patients with rheumatoid arthritis and low disease activity. Ann Rheum Dis 2004; 63: 31-35.

9. Gradus-Pizlo I, Sawada SG, Wright D, Segar DS, Feigenbaum $\mathrm{H}$. Detection of subclinical coronary atherosclerosis using twodimensional, high-resolution transthoracic echocardiography. $J$ Am Coll Cardiol 2001; 37: 1422-1429.

10. Weyman AE, Feigenbaum H, Dillon JC, Johnston KW, Eggleton RC. Noninvasive visualization of the left main coronary artery by cross-sectional echocardiography. Circulation 1976; 54: 169-174.

11. Rink LD, Feigenbaum H, Godley RW, Weyman AE, Dillon JC, Phillips JF, et al. Echocardiographic detection of left main coronary artery obstruction. Circulation 1982; 65: 719-724.

12. Vered Z, Katz M, Rath S, Har-Zahav Y, Battler A, Benjamin P, et al. Two-dimensional echocardiographic analysis of proximal left main coronary artery in humans. Am Heart J 1986; 112: 972-976.

13. Petrovic O, Elsner GB, Wilensky RL, Swanson ST, Feigenbaum H. Transthoracic echocardiographic detection of coronary atherosclerosis. Am J Cardiol 1996; 77: 569-574.

14. Arnett FC, Edworthy SM, Bloch DA, McShane DJ, Fries JF, Cooper NS, et al. The American Rheumatism Association 1987 revised criteria for the classification of rheumatoid arthritis. Arthritis Rheum 1988; 31: 315-324.

15. Glagov S, Weisenberg E, Zarins CK, Stankunavicius R, Kolettis GJ. Compensatory enlargement of human atherosclerotic coronary arteries. N Engl J Med 1987; 316: 1371-1375.

16. Wada N, Watanabe N, Yamaura Y, Neishi Y, Koyama $\mathrm{Y}$, Kawamoto $\mathrm{T}$, et al. Comparison of high-frequency two-dimensional transthoracic echocardiography versus intravascular ultrasound for evaluation of the left anterior descending coronary artery. Am J Cardiol 2005; 96: 1746-1749.

17. Takeuchi M, Yoshitani H, Miyazaki C, Yoshikawa J. Relationship between the number of coronary risk factors and coronary atherosclerosis assessed by high-frequency transthoracic echocardiography. J Am Soc Echocardiogr 2006; 19: 1056-1062.

18. Gradus-Pizlo I, Feigenbaum H. Imaging of the left anterior descending coronary artery by high-frequency transthoracic and epicardial echocardiography. Am J Cardiol 2002; 90: 28L-31L.

19. Ogeng'o JA, Kilonzi JP, Mwachaka PM, Ogeng'o NM, Misiani MK, Murunga A. Intima - media thickness of left anterior descending coronary artery in a black kenyan population: correlation with morphological features. Anat Physiol 2014; 5 : 1.

20. Gradus-Pizlo I, Bigelow B, Mahomed Y, Sawada SG, Rieger $\mathrm{K}$, Feigenbaum H. Left anterior descending coronary artery wall thickness measured by high-frequency transthoracic and epicardial echocardiography includes adventitia. Am J Cardiol 2003; 91: 27-32.

21. Hausmann D, Friedrich G, Soni B, Daniel WG, Fitzgerald PJ, Yock PG. Validation of Automated Border Detection in Intravascular Ultrasound Images. Echocardiography 1996; 13: 599-608. 